\title{
SENSITIVITY ANALYSIS OF THE AHP VS A FUZZY DECISION METHODOLOGY
}

\author{
Fatemeh Ghotb and Lewis Warren \\ Swinburne University of Technology, PO Box 218, Hawthom Vic 3122, Australia \\ fghotb@banyan.swin.edu.au
}

\begin{abstract}
In this paper the Analytical Hierarchy Process and a Fuzzy Decision method were applied to the question of whether to introduce a new IT system into a public hospital. Both, the same factors and the same experts for rating, were used when applying the methods. The results and practical aspects of using the two methods are compared. The sensitivity of the two methods with respect to changes in ratings was studied.
\end{abstract}

\section{INTRODUCTION}

A decision methodology can be considered as good as its output results. If different approaches lead to different conclusions, the reasons for the divergence need to be analysed so that the appropriate method can be selected to fit the decision situation in question. The Analytic Hierarchy Process(AHP) and Fuzzy Decision Analysis(FDA) were selected for comparison since this application required a practical method that was easy to apply and easy to explain. Both methods satisfied these conditions.

This hospital case was considered an appropriate medium for comparison since it had many qualitative aspects. Building extensions to the hospital had raised the question of whether to relocate the existing method for storing radiological medical images on film, or whether to introduce a completely new system based on digital imaging technology. These two alternatives are termed as Old System and New System, and the two methodologies attempt to compare the overall benefits to the hospital associated with each system. The following aspects of the application were compared:

1. The relative level of preference for each alternative.

2. The sensitivity of the methods to possible changes in hospital policy represented as different weights for the major factor groups.

3. The rating difficulty associated with the different methods. Both methods elicit expert opinion for rating. The AHP uses pairwise factor comparisons with a numeric or linguistic scale. The FDA rates factors in isolation and only uses a linguistic scale. The accuracy of the rating method was not evaluated in this study. To evaluate how accurately each method represented the raters true feelings, an experiment for that specific purpose would be required. Although the raters had opinions regarding the difficulty of rating, opinions on accuracy were not clearly formed.

To facilitate the comparison it was necessary to make certain assumptions regarding the New System. There were several options to address in the specification and configuration of a new digital system. The selection between these options is another problem again but for the purposes of this study one specification was decided upon which represented a realistic system for the hospital to start with. Vendor information provided the basis for costing all aspects over various lifetime estimates. Furthermore, in order to rate the relative performance of the various aspects of the two alternatives, actual knowledge of the New System performance for radiological diagnosis is required as distinct to vendor claims. The medical experts used for rating did have such experience and the fact that it was not suitable for certain types of radiological diagnosis was allowed for. The software and hardware required for such a New System was also 
assumed to have developed to a satisfactory level of technical reliability although some new types of risks introduced were evaluated.

The Goal was to maximise the overall benefits to the hospital considering all costs and intangible tradeoffs. In order to achieve this goal, a preference measure or benefit index(BI) was derived for each alternative and for comparison purposes a new index was defined called the New System Superiority Margin (SSM) where ;

$$
\text { SSM }=\frac{\text { New System BI - Old System BI }}{\text { Old System BI }}
$$

The SSM as a percentage, is used to compare the results of the two methodologies. With the AHP the benefit index (BI) was the overall AHP priority values for each alternative, and with the FDA the BI used in the above equation was a calculated ranking index for each of the membership sets.

\section{THE DECISION STRUCTURE}

In order to evaluate each method's sensitivity to possibie changes in hospital policy and attitudes, it was decided to form major groups of factors that would be similarly affected by policy changes. Three groups were decided as sufficient to capture fundamental policy changes. These three groups were Costs, Productivity and Risks. All decision factors were thus divided into these three main groups and nine different 'policies representing the varying importance of each of these groups were applied. In this way, the comparison was effectively of a three level decision problem - Goal, Main Groups, and Alternatives. However, within each main group the two methods processed and aggregated the ratings in different manners. To enable the results to be compared, consistency between method applications was achieved by using the same raters, the same main groups of factors, the same decision factors and the same policy sets. The major groups of factors were:

Costs: A public hospital must be highly conscious of all costs since funding is traditionally very tight with little chance of finding new revenue sources. For these system alternatives all lifetime costs were modeled by spreadsheet and reduced to their Present Value and Annual Equivalent Cost. Possible future variations in discount factor, escalation and lifetime length were considered. The Annual Equivalent Cost was used as input to both decision methods. The Cost priorities calculated by the AHP were derived from ratios of the two system cost values. The Annual Equivalent Costs resulting from the most likely future scenario were used in this calculation. With the FDA it was possible to represent the Annual Equivalent Costs for a range of future scenarios as one fuzzy set. This was then normalised to enable aggregation with the qualitative ratings.

Productivity: This group of factors contained all those factors related to productivity which could not be dollar quantified in the time available, but which could be performance estimated by the experts. With changes to funding, productivity may become more important. So the different policies reflect different management attitudes towards the productivity group.

Risks: Being a public service organisation the hospital must provide a service with a high degree of reliability. This responsibility requires the hospital to examine any new risks introduced over those that traditionally exist. In terms of relative importance, the management attitude towards risk may also change over time by virtue of market competition and the need to become more progressive. 


\section{THE AHP APPLICATION}

The AHP has been widely applied and a detailed explanation of the method can be found in several sources $[8,9]$ and a good selection of case studies in $[4,1]]$. A rating scale of 1 to 9 is generally used to reflect the relative preference of one factor over another in pairwise comparisons. Both, performance rating for the decision alternatives and the relative factor importance, are evaluated in this manner. The overall importance of the decision factors on the various levels are then derived by proportioning through the hierarchy. For each comparison matrix of similar level factors, the priorities are derived from the matrix eigenvalues. Figure 1 shows the overall hierarchy of the problem.

\section{Cost Priority Calculation:}

The Annual Equivalent Costs (AEC) of the two alternatives for the most likely scenario were:

$\mathrm{AEC}$ (Old System) $=\$ 569,578$

$\mathrm{AEC}$ (New System) $=\$ 899,567$

Let AECR represent the Annual Equivalent Cost Ratio which is calculated as follows:

$$
\mathrm{AECR}=\frac{\mathrm{AEC}(\text { Old System })}{\mathrm{AEC}(\text { NewSystem })}=0.633
$$

Then

$$
\begin{aligned}
& \text { Cost Priority(Old System) }=\frac{1}{1+\text { AECR }}=0.613 \\
& \text { Cost Priority(New System) }=\frac{\text { AECR }}{1+\text { AECR }}=0.387
\end{aligned}
$$

Productivity and Risk Priority Calculation:

Global synthesis over the factor hierarchy was achieved with aid of the software package Expert Choice. Table 1 shows the synthesized priorities for each alternative for the Productivity and Risk groups of factors as well as the Cost priorities derived above. These values were later combined according to the 9 different policies and the New System Superiority Indices calculated for comparison with the FDA results. 


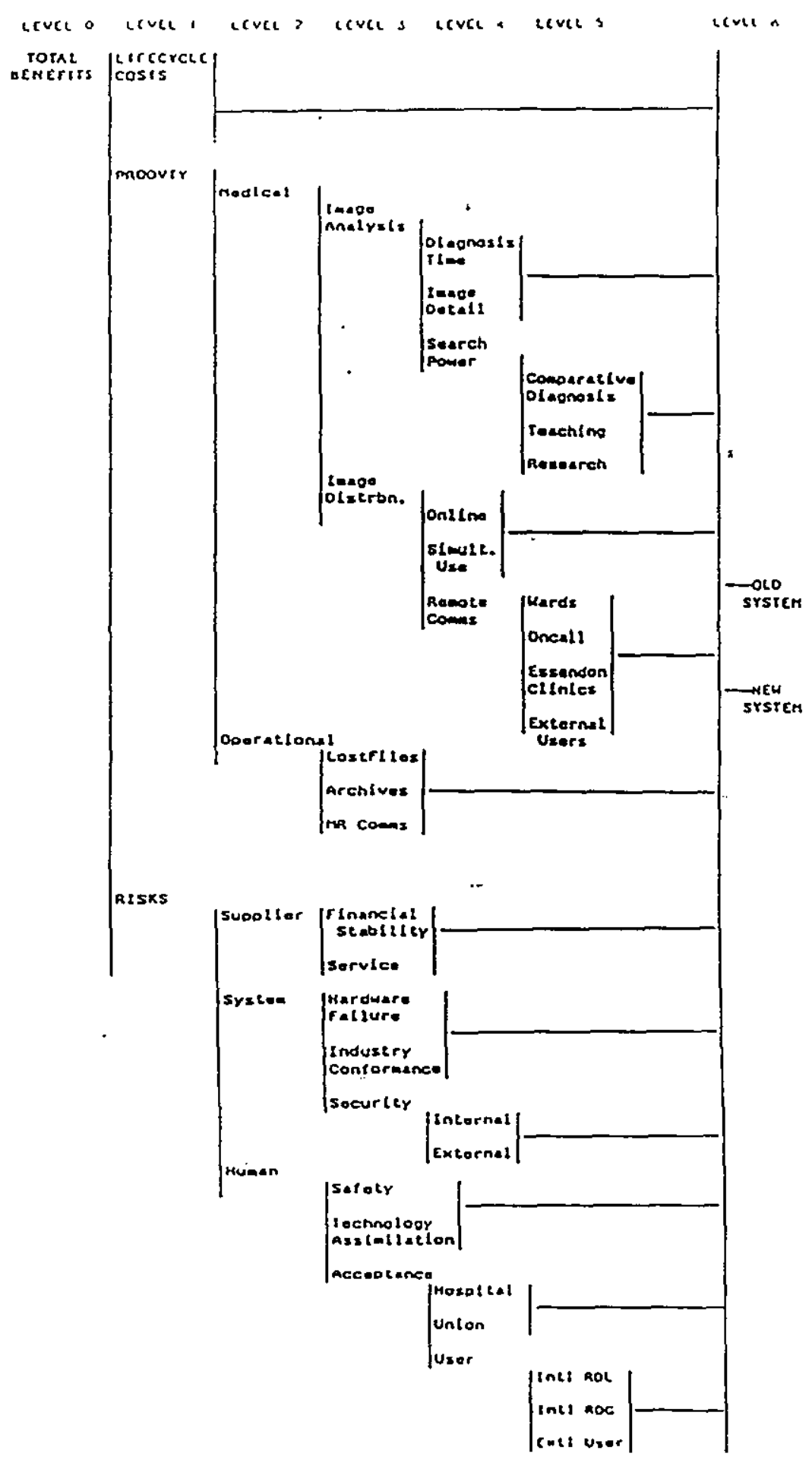

Figure 1. The Herarchy of the AHP Model 
Table 1 Overall Group Priorities - AHP

\begin{tabular}{|l|l|l|l|}
\hline Altemative & Productivity & Risks & Costs \\
\hline Old system & 0.117 & 0.688 & 0.613 \\
New System & 0.883 & 0.312 & 0.387 \\
\hline
\end{tabular}

\section{THE FUZZY DECISION ANALYSIS}

A detailed explanation of this method can be found in [6] using a hypothetical plant location application. The same authors also apply the method to personnel selection [7]. No hierarchical structure of factors is required with this method which uses linguistic variables for rating of qualitative factors and fuzzy set representations. Quantitative factors are also represented as fuzzy membership sets which show the degree of belief that a variable will take any particular value. The membership sets for the quantitative factors need to be normalised before they can be aggregated to those representing ratings for the qualitative factors. For each of the three major groups of factors an aggregate fuzzy set represents the effective performance of each alternative. These are then combined by the group or policy weights to form a membership set representing the overall performance of the alternative with respect to the goal. Finally, a ranking method is applied to represent the 'utility' of the membership sets for the two alternatives. This study follows the Chen ranking procedure [3], and the ranking index is used a measure for total benefits or as a benefit index(BI). An explanation of fuzzy set theory is beyond the scope of this paper however the linguistic rating variables and their membership set representations are shown in Figures 2 and 3.
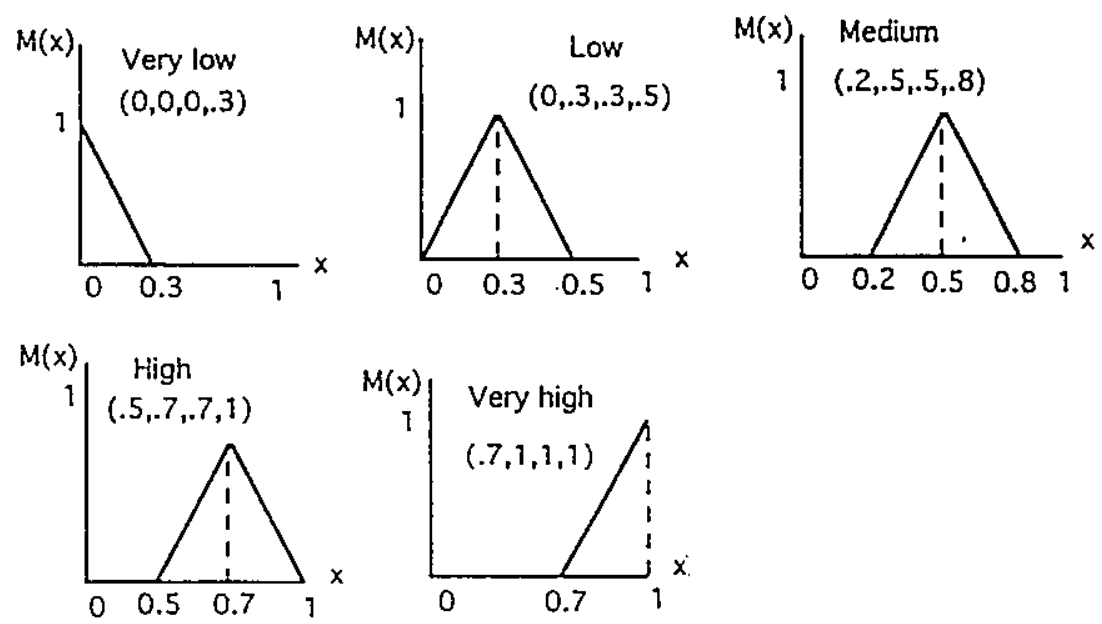

Figure 2. The Linguistic Rating Variables 

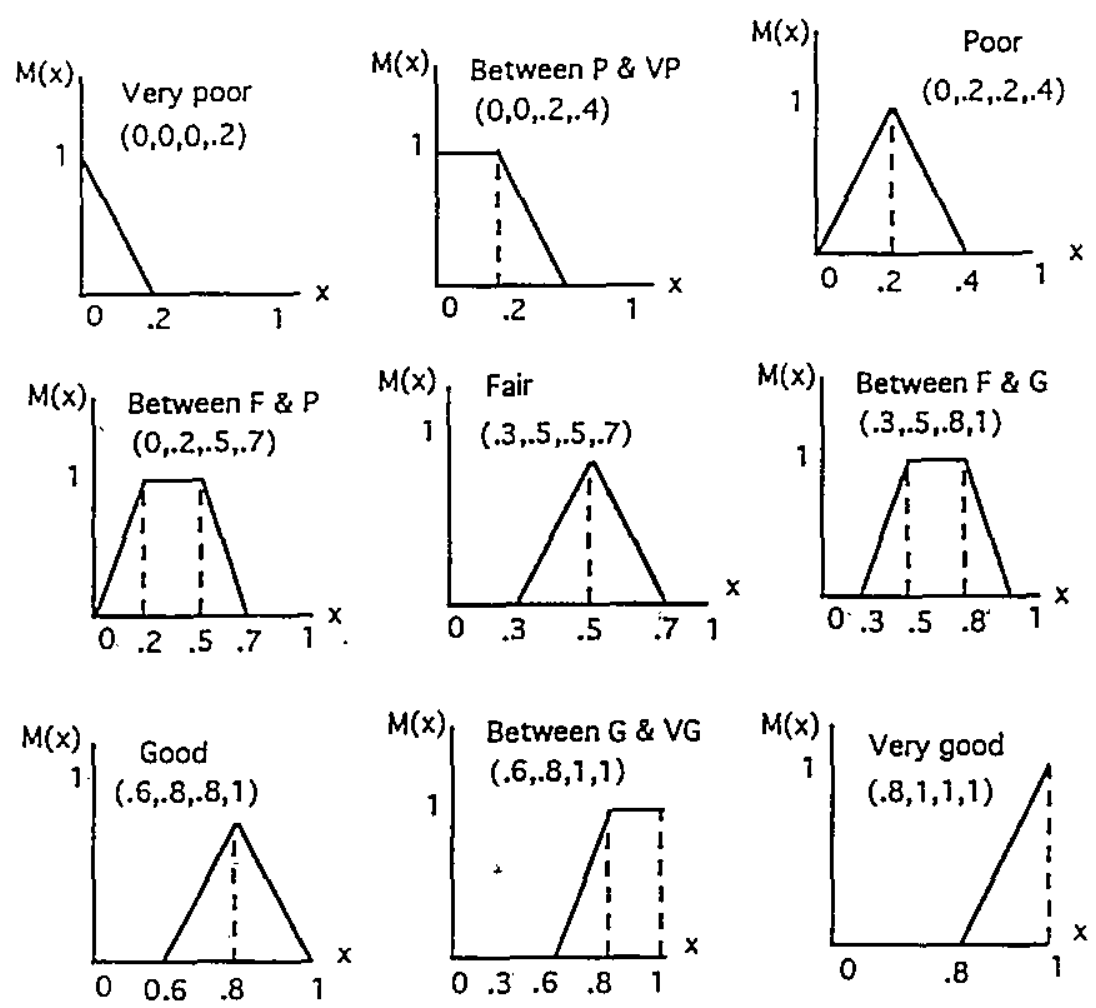

Figure 3. The Fuzzy Membership Sets

Costs: The range of Annual Equivalent Costs (AEC) determined by spreadsheet modelling was represented as a trapezoidal membership set. The extremities are the minimum and maximum values while the two intermediate points are the most likely range (Figure 4). These sets are then normalised to translate the possible variable range to the interval 0 to 1 .

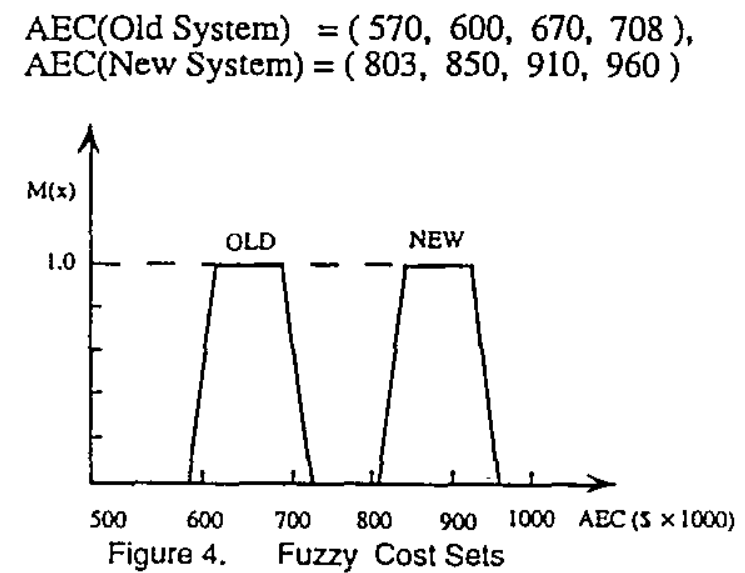

Productivity and Risks: Linguistic rating sets are already on the 0 to 1 scale, so all these qualitative factors can be weighted and aggregated directly for each group for each alternative. The groups' aggregate values are shown in Table 2. 
Table 2. Aggregated Values for Productivity and Risk

\begin{tabular}{|l|c|c|}
\hline Alternative & Productivity & Risk \\
\hline Old System & $.06, .18, .02, .44$ & $.34, .65, .66, .81$ \\
New System & $.33, .65, .65, .88$ & $.29, .60, .61, .85$ \\
\hline
\end{tabular}

The Overall Performance: The final membership set $(F)$ after combining the three groups represents the overall performance of the alternative towards achieving the Goal. Figure 5 shows the final sets derived for each alternative using the policy number one.

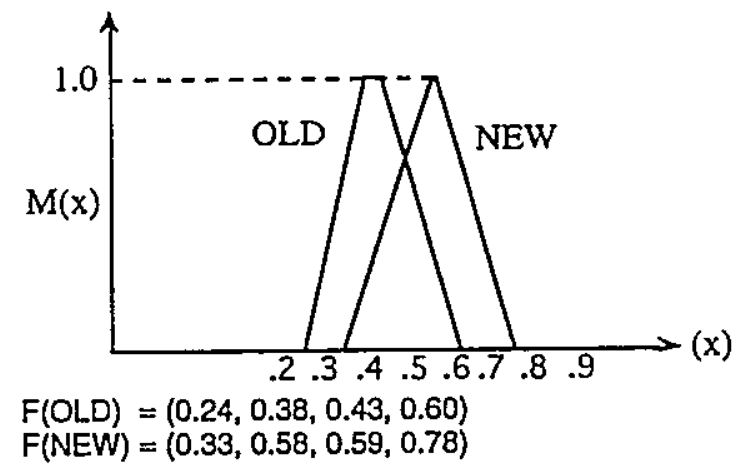

Figure 5. Overall Performance Under Policy No. 1

Ranking Index: The Chen ranking method[3], uses maximising and minimising sets to calculate a crisp index for a relative comparison of the two sets. The System Superiority Margin (SSM) is derived from these indices.

\section{SENSITIVITY ANALYSIS}

A sensitivity analysis usually investigates the changing contribution that a factor makes to the overall priorities as its importance(weight) ranges from 0 to $100 \%$. The factors of interest are the top level factors which in this study are Costs, Productivity and Risks. Single factor analysis varies the importance of one factor while weighting the other factors equally. Policies 1 to 4 are examples of this type of weight distribution (Table 3 ). The AHP software, EXPERT CHOICE, develops such factor sensitivity graphs. However, for the purpose of method comparison, we are more interested in method sensitivity than factor sensitivity and consequently graphs of top level factor sensitivity are not of prime interest.

A new aspect is now introduced whereby fuzzy membership sets are used to model policy statements which represent relative group weights (Table 4, Policies 5- 9). A fuzzy set derived from a policy statement is now used to show the degree of possibility that any weight value will be applied to the top level factor aggregation. This is distinct to the fuzzy linguistic variable sets used to aggregate the subfactors. Fuzzy sets appear to possess a great facility for representing policy statements which detail overlapping tradeoffs, so we decided to test several for top level aggregation in both decision methods. The decision methods' sensitivities were evaluated by examining the variations in SSM over the nine policies tested (Tables 3 and 4) 
Table 3. Policy Definitions with Crisp Group Weights

\begin{tabular}{|l|l|l|l|l|}
\hline $\begin{array}{l}\text { Policy } \\
\text { No. }\end{array}$ & $\begin{array}{l}\text { Policy Description } \\
\text { - Cost }\end{array}$ & Productivity & Risk \\
\hline 1 & $\begin{array}{l}\text { Strong Productivity } \\
\text { Emphasis }\end{array}$ & 0.25 & 0.50 & 0.25 \\
3 & Equal Emphasis & 0.33 & 0.33 & 0.33 \\
4 & $\begin{array}{l}\text { Strong Cost } \\
\text { Emphasis }\end{array}$ & 0.50 & 0.25 & 0.25 \\
& $\begin{array}{l}\text { Strong Risk } \\
\text { Emphasis }\end{array}$ & 0.25 & 0.25 & 0.50 \\
\hline
\end{tabular}

Table 4. Policy Definitions with Fuzzy Group Weights

\begin{tabular}{|c|c|c|c|c|}
\hline $\begin{array}{l}\text { Policy } \\
\text { No. }\end{array}$ & Policy Description & Cost & Productivity & Risk \\
\hline 5 & $\begin{array}{l}\text { Slight risk emph. \& } \\
\text { Prod. de-emph }\end{array}$ & $.23, .28, .38, .43$ & $.18, .28, .38, .43$ & $.23, .28, .38, .48$ \\
\hline 6 & $\begin{array}{l}\text { Slight Cost \& Risk } \\
\text { Emphasis }\end{array}$ & $.23, .28, .38, .48$ & $.23, .28, .38, .43$ & $.23, .28, .45, .50$ \\
\hline 7 & $\begin{array}{l}\text { Slight Risk } \\
\text { Emphasis }\end{array}$ & $.30, .33, .33, .36$ & $.30, .33, .33, .36$ & $.30, .33, .33, .42$ \\
\hline 8 & $\begin{array}{l}\text { Slight Prod. } \\
\text { de-Emphasis }\end{array}$ & $.30, .33, .33, .36$ & $.24, .33, .33, .36$ & $.30, .33, .33, .36$ \\
\hline 9 & $\begin{array}{l}\text { Strong Risk emph. } \\
\text { \& Cost de-emph. }\end{array}$ & $.21, .26, .33, .33$ & $.30, .33, .33, .36$ & $.33, .33, .40, .45$ \\
\hline
\end{tabular}

The assignment of the fuzzy sets is arbitrary but the above examples show how the trapezoidal membership sets may be shifted or skewed to represent subtle variations in policy. For example, a full expression of Policy 6 could be :

"A possible small deviation around equal weighting for all groups, but with a slight emphasis on Costs and a slightly greater emphasis on Risk avoidance."

Table 5. Summary Results for Crisp Weight Policies

\begin{tabular}{|c|l|l|l|l|l|l|}
\hline \multirow{2}{*}{ Policy } & $\begin{array}{l}\text { AHP } \\
\text { Priority }\end{array}$ & \multirow{2}{*}{ SSM } & \multicolumn{2}{|l|}{$\begin{array}{l}\text { FDA } \\
\text { Ranking Index }\end{array}$} & \multirow{2}{*}{ SSM } \\
\cline { 2 - 3 } \cline { 5 - 6 } & Old Sys. & New Sys. & & Old Sys. & New Sys. & \\
\hline 1 & .381 & .619 & $+62 \%$ & .345 & .585 & $+70 \%$ \\
2 & .470 & .530 & $+13 \%$ & .445 & .555 & $+25 \%$ \\
$3^{*}$ & .501 & .499 & $-0.4 \%$ & .510 & .525 & $+3 \%$ \\
$4 *$ & .523 & .477 & $-9 \%$ & .445 & .520 & $+17 \%$ \\
\hline
\end{tabular}


Table 6. Summary Results for Fuzzy Weight Policies

\begin{tabular}{|c|c|c|c|c|c|c|}
\hline Policy & $\begin{array}{l}\text { AHP } \\
\text { Ranking }\end{array}$ & & SSM & $\begin{array}{l}\text { FDA } \\
\text { Ranking }\end{array}$ & & SSM \\
\hline & Old Sys. & New Sys. & & Old Sys. & New Sys. & \\
\hline $\begin{array}{l}5 \\
6 \\
7 \\
8 \\
9\end{array}$ & $\begin{array}{l}.390 \\
.415 \\
.425 \\
.360 \\
.410\end{array}$ & $\begin{array}{l}.535 \\
.525 \\
.520 \\
.575 \\
.595\end{array}$ & $\begin{array}{l}+37 \% \\
+27 \% \\
+22 \% \\
+60 \% \\
+45 \%\end{array}$ & $\begin{array}{l}.400 \\
.420 \\
.400 \\
.420 \\
.420\end{array}$ & $\begin{array}{l}.475 \\
.490 \\
.500 \\
.520 \\
.525\end{array}$ & $\begin{array}{l}+19 \% \\
+17 \% \\
+25 \% \\
+24 \% \\
+25 \%\end{array}$ \\
\hline
\end{tabular}

\section{COMPARISON OF RESULTS}

It should be noted that the Chen ranking indices do not need to sum to unity whereas the AHP priorities do. Generally, the results showed a fair degree of similarity (Tables 5 and 6). Only two policies showed a SSM deviation of greater than $25 \%$ between methods and although a different alternative was chosen to be superior in two cases, one was only marginally different (policy No. 3 ). The maximum SSM deviation between methods was $36 \%$ for policy 8 and each method showed about half SSM's above and half below the other.

\section{COMPARISON OF METHODS}

\section{The Analytic Hierarchy Process}

\section{Advantages:}

- Forces the decision maker to think about comparisons they might not make otherwise

- The hierarchy helps to formulate the decision in a logical way

- Highlights inconsistent ratings

- Well designed software is available

Disadvantages:

- Pairwise comparisons are sometimes difficult and tedious

- A software limit on the number of Hierarchy levels that can be used

\section{Fuzzy Decision Analysis}

\section{Advantages:}

- There is no limit on the number of factors and the complexity of analysis is not greatly affected by the number of factors

- Rating was generally considered easier by the raters

- No unusual or difficult comparisons due to individual factor ratings

- A large selection of every day words to express the exact rating feeling

- The membership set concept allows a large amount of information to be represented in a single construct for logical or algebraic manipulation.

\section{Disadvantages:}

- Inconsistent ratings are not detected

- The ranking method is critical in the decision process and there is no single ranking method that is generally considered to be superior. 


\section{CONCLUSIONS}

The results from the methods were very similar and both methods were considered to be useful for approaching this complex decision in a systematic way. On the basis of this study, neither is obviously superior. However, the Fuzzy Decision Analysis does seem to inherently be very powerful by virtue of the ease with which Fuzzy Theory can deal with all kinds of uncertainties. This ability would seem to lead naturally to management aids which integrate decision tools with learning systems.

Various avenues for further research present themselves. Two obvious areas are to apply other ranking methods with the FDA and to apply other rating scales with the AHP analysis. The authors are currently pursuing both these comparisons. But more importantly, there is a need for further case studies of the FDA which compare output results with those of other decision methodologies.

\section{References}

1. Arbel A. and Seidmann A. (1984), 'Performance Evaluation of Flexible Manufacturing Systems', IEEE Trans. Systems, Man, Cybemetics Vol. SMC-14, No. 4 pp 606-617.

2. Bernard R. H. and Canada J. R. (1990), 'Some Problems in Using BenefiUCost Ratios with the Analytic Hierarchy Process', The Engineering Economist, Vol. 36, No. 1.

3. Chen S. H. (1985), ' Ranking Fuzzy Numbers', Fuzzy Sets and Systems, Vol. 17. pp /13-129.

4. Golden B. et al. eds (1989). 'The Analytic Hierarchy Process' Spring Verlag, Heidelberg

5. Kandel A. (1986), 'Fuzzy Mathematical Techniques with Applications', Addisin Wesley, NY

6. Liang G. and Wang M. (1991), 'A Fuzzy Multi-criteria Decision making method for Facility Site Selection', Int. J. Prod. Res., Vol. 29, No. 11., pp 2313 - 2330

7. Liang G. and Wang M. (1992), 'Personnel Selection Using a Fuzzy Multicriteria Decision Method', Proc. Joih Intl. Conf. MCMD, Taipei, Vol.3, pl

8. Saaty T. L. (1980), 'The Analytic Hierarchy Process' , McGraw--Hill, New York.

9. Saaty T. and Kearns K. (1985) 'Analytical Planning', Pergamon Press., Elmsford, NY.

11. Zahedi F. (1986), The Analytic Hierarchy Process-A Survey of the Method and its Applications', Interfaces, July- Aug(pp 96-108).

12. Zimmermann H. J. et al. (1991), 'Fuzzy Sets and Decision Analysis', North Holland, Studies in Mgt. Sci., Vol. 20. 